\title{
XLVI. On a method of examining the divisions of astronomical instruments. In a letter to the Rev. Dr. Maskelyne, F.R.S. Astronomer Royal
}

\section{Rev. William Lax A.M. F.R.S.}

To cite this article: Rev. William Lax A.M. F.R.S. (1810) XLVI. On a method of examining the divisions of astronomical instruments. In a letter to the Rev. Dr. Maskelyne, F.R.S. Astronomer Royal , Philosophical Magazine Series 1, 35:144, 277-286, DOI: 10.1080/14786441008563081

To link to this article: http://dx.doi.org/10.1080/14786441008563081

Published online: 18 May 2009.

Submit your article to this journal $\pi$

山 Article views: 2

View related articles 
c. Rectangular, a particular name given to the staurotide composed of two prisms which cross at right angles.

d. Obliqueangled, a particular name given to the staurotide composed of two prisms which cross at an angle of $60^{\circ}$.

e. Sexradiated, a name given to the staurotide composed of three prisms which cross so as to represent the six radii of a regular hexagon.

$f$. Cruciform, a name given to the harmotome composed of two crystals which form a kind of cross.

g. Triglyphous, when the strix considered on three faces united around one and the same solid angle, are in three directions perpendicular to each other. Ex. Triglyphous sulphurated iron.

h. Geniculated, when it is composed of two prisms which unite by one extremity forming a kind of knee. Ex. Geniculated oxidated titanium.

In the descriptions of the species we shall meet with a small number of denominations which we have here omitted. But their signification will be immediately obvious, or will resolve itself in to that of some of the foregoing denominations.

[To be continued.]

XLVI. On a Method of examining the Divisions of astronomical Instruments. By the Rev. William Lax, A.M., F.R.S., Lowndes's Professor of Astronomy in the University of Cambridge. In a Letter to the Rev. Dr. Maskety Ne, F.R.S. Astronomer Royal*.

Dear Sir, I Am persuaded that you must feel, in common with myself, how unpleasant it is to make use of an in. strument in astronomical observations requiring extreme accuracy, whose exactness you have no adequate means of ascertaining, but are obliged to depend for it in a great measure upon the abilities and integrity of the artist. It is in vain that we observe with so much nicety, and readoff with so much precision, if we are still uncertain whether there may not be an error in the instrument itself of much greater magnitude than those which we are endeavouring to prevent; and that our best instruments must be liable to such errors, no person can possibly doubt, who has paid due attention to the sources from whence they may arise. I have estimated, as accurately as I could, the amount to which they may accumulate in Bird's method of dividing by continual bisections, and have satisfied myself that they are much more considerable than is generally

* From the Philosophical Transactions for 1809 , Part II. 
apprehended : but as I cannot obtain such precise information as I could wish, respecting the exactness with which a bisection can be performed, or a length taken from the scale of equal parts and laid upon the instrument, I will not trouble you with the deduction which $I$ have made. It is understood, indeed, that Bird's method is now generally laid aside, and that each artist employs one, which he considers in many respects as peculiar to himself; but I presumed that there would still be such a connexion betwixt Bird's method and those which have been substituted in its stead, as to render them in some degree liable to the same errors to which it was subject; and the reports which I have uniformly received from persons, who have had an opportunity of examining some of the modern instruments, have fully convinced me that my opmion was just. But whatcver may be the nature of the methods which are now in use, or whatever their advantages over Bird's, I never conld persuade myself that it would be safe to trust to an instrument, without a previous examination. To discover the means of accomplishing this object, is what $I$ have for some time bean anxious to effect; and though I fear my endeavours have not been very successfil, I will nevertheless take the liberty of presenting you with the result.

You are aware, I believe, that I use a circular instrument for observing both in altitude and azimuth, which was made for me bv Mr. Carey in the Strand; that the radius of both the altitude and the azimuth circle is one foot, and that each is divided into parts containing ten minutes. The construction of this instrument does not differ materially from that of other similar instruments, with which you are well acquainted, and I shall not therefore waste your time by giving you a particular description of it. For the purpose of examining the divisions upon the two circles, I procured an apparatus to be prepared by Mr. Carey, which will be very easily explained. To the face of the rim which surrounds the azimuth circle, and with its left end close to the stand which supports the micrometer on the east side, an arc of brass, concentric with the circle itself, and a little more than $90^{\circ}$ in length, an inch in breadth, and one eighth of an inch in thickness, is firmly fixed by screws, so as to have the plane parallel to the plane of the circle, and a small portion of its lower surface resting upon the extreme part of the rim. The szrews pass through a brass arc, which is fastened to this at right angles, and lies with its broad side against the face of the rim. Upon the first-mentioned arc, a strong upright piece of brass, about 
six inches in length, is made to slide, the lower part of it embracing the arc as a groove, and having a clamping screw underneath, for the purpose of fixing it firmly to the are at any point required. To the top of the upright piece of brass is attached a microscope, with a moveable wire in its focus, pointing down to the division upon the circle, not directly, however, but with an inclination to the left of about $30^{\circ}$. This inclination is given to it, in order to make it point to the same division upon the circle, which is immediately under the micrometer itself, when it has been moved up as near to the micrometer as it is capable of approaching. The microscope has attached to it a small graduated circle of brass, and an index, by which the seconds, and parts of a second, moved over by the wire are determined.

To the vertical circle there is likewise an arc applied, of the same length and breadth as the former, but somewhat thicker, and of a radius exceeding that of the circle by about two inches. This greater thickness is given to it, on account of its being supported in a manner which renders additional strength necessary. It is fixed with its broad convex side downwards upon two brass pillars, screwed fast to the plane of the azimuth circle, and standing in a line parallel to the plane of the vertical circle at the distance of about four inches from it, and on the right side of the pillars which support the micrometers belonging to this circle. The pillar, to which the left end of the arc is fastened, is placed close to the lower micrometer of the vertical circle, and the other contiguous to the elevated rim, in which the divisions of the azimuth circle are cut. The right end of the arc reaches beyond this pillar about ten inches. The pillars are of such a height, and so proportioned to each other, that whilst the $\mathrm{kft}$ end of the arc, which lies horizontally, is raised to within about two inches of the height at which the lowest point of the vertical circle is placed, the whole arc runs parallel to the circle through an extent of something more than $90^{\circ}$. Upon the arc a microscope, with a moveable wire in the focus, is made to slide as in the former case, and to point to the divisions upon the vertical circle, not directly, but with an inclination of about $30^{\circ}$ to the left, in order that the same division (which is the lowest upon the circle) may be scen through it and through the lower micrometer at the same time.

I will now proceed to show you in what manner the examination of the divisions upon either circle may be 
performed. The process is precisely the same in both cases, and will of course be described in the same words.

The first point to be exammed is that of $180^{\circ}$, which must be done in the usual way, by bringing the points of 0 and $180^{\circ}$ to the moveable wires of the opposite micrometers, and then turning the circle half-way round, and bisecting the points again with the maveable wires; and lastly, taking half the difference betwixt the distances of the wires in the two positions of the circle for the error at the point of $180^{\circ}$. Having now bisected the noint of zero with the moveable wire of the micrometer, which is intended to be used in the rest of the process (for we shall have no further occasion for both), we must slide the microscope alung the arc, till by moving the wire a little we can bisect the point of $90^{\circ}$, and then the micrometer must be firmly clamped to the arc. The circle must then be turned till the point of $180^{\circ}$ is brought to the microscope, and that of $90^{\circ}$ to the micrometer, so that we may be able to bisect each by a slight motion of their respective wires. This being done, we must observe, from the positions of the wires, how much the interval betwixt them has increased or decreased in the measurement of the new arc; and this increase or decrease must be noted down with a t or - accordingly. In the same manner we must proceed through the remaining two ares of $90^{\circ}$, observing and noting down the difference betwixt each and the original arc.

The point of zero must now be brought again to the micrometer, and bisected by the moveable wire, and the microscope be made to slide back along the arc, till by moving the wire a little we can bisect the point of $60^{\circ}$; and when this is done, the microscope must be clamped. We must then measure the arc of $60^{\circ}$ against every succeeding arc of $60^{\circ}$ in the circle, precisely in the same way that we measured the first are of $90^{\circ}$ against the other three. The arc of $45^{\circ}$ is next to be measured against every succeeding arc of $45^{\circ}$, and this will complete all that is necessary to be done in the early part of the morning before the heat of the sun can have affected the temperature of the instrument. The rest may be performed at our leisure.

You will immediately perceive the object of this kind of measurement. It enables us to determine, with any degree of accuracy that may be required, the proportion which the first and every succeeding arc of the circle, contained betwixt the micrometer and the microscope, bears to the whole circle, and of course the absolute length of 
the arcs themselves. Let $a$ denote the real length of the first of these, and $\pm a^{\prime}, \pm a^{\prime \prime}, \pm a^{\prime \prime \prime}, \& c$., the difference betwixt the first and second, the first and third, \&c. respectively; let $A$ represent any other arc whose length is known, and which is a multiple of $a$, as marked upon the instrument, and let this multiple be expressed by $n$. Then will $a+\left(a+a^{\prime}\right)+\left(a+a^{\prime \prime}\right)+\left(a+a^{\prime \prime \prime}\right)+8 \mathrm{c} . .$. $\left(a+a^{\prime \prime \prime n^{n-1}}\right)=\mathrm{A}$, and $a=\frac{\mathrm{A}-a^{\prime}-a^{\prime \prime}-\ldots a^{\prime \prime n^{n-1}}}{n}$. Hence it is evident, that if there is no error committed in the measurement of any of these arcs, we shall have the value of $a$, and consequently of $a+a^{\prime}, a+a^{\prime \prime}, a+a^{\prime \prime \prime}, \& c_{n}$, and of any arc, comprehending any number of these, accurately determined. But if there be an error of $e$ in the measurement of the first, of $e^{\prime}, \epsilon^{\prime \prime}, e^{\prime \prime \prime}, \& c_{.}$, in the measurement of the second, third, \&c., respectively, then we shall have the following equation for determining $a$, viz. $a+\left(a+a^{\prime}\right.$ $\left.+e+e^{\prime}\right)+\left(a+a^{\prime \prime}+e+e^{\prime \prime}\right)+\& \mathrm{c} . \ldots . .\left(a+a^{\prime \prime \prime} \cdot \overline{x-1}+e\right.$ $\left.+\epsilon^{\prime \prime \prime^{n-1}}\right)=\mathrm{A}$, and consequently $a$ will appear to be equal to $\frac{A-a^{\prime}-a^{\prime \prime}-\ldots a^{\prime \prime \prime} \cdot \overline{n-1}}{n} \overline{n-1} e-e^{\prime}-e^{\prime \prime}-\ldots e^{\prime \prime \ldots . . \overline{n-1}}$, which

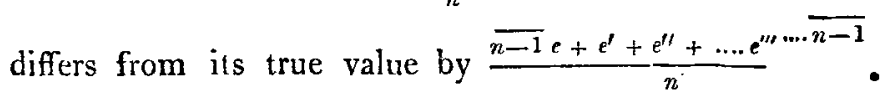
Hence it follows, that the value of the $p^{\text {th }}$ are ( $p$ being greater than unity), as deduced by this process, will differ from its true value by $\frac{\overline{n-1} \cdot e+e^{\prime}+e^{\prime \prime}+\ldots e^{\prime \prime \prime} \ldots \overline{p-1}+e^{\prime \prime \prime \ldots p}+\ldots e^{\prime \prime \prime} \cdot \cdot n-1}{n}$ $-e-e^{\prime \prime \prime \cdots \overline{p-1}}$, and that if we add any number $p$ of these arcs together, in order to determine the value of the arc which is cqual to their sum, we shall have an error in this value (and the expression holds when $p$ is unity, or the first arc only is taken) equal to $p \frac{\overline{n-1} \cdot e+e^{\prime}+e^{\prime \prime}+\ldots e^{\prime \prime} \cdot \overline{p-1}}{n}$ $\underbrace{+e^{\prime \prime \prime \cdots . . p}+\ldots e^{\prime \prime \prime} \overline{n-1}}_{n}-\overline{p-1} \cdot e-e^{\prime}-e^{\prime \prime}-\ldots e^{\prime \prime \prime \cdots \overline{p-1}}=\overline{n-p}$. $\overline{\frac{e^{\prime}-e^{\prime \prime}-\ldots e^{\prime \prime \prime} \cdots \overline{\overline{p-1}}}{p+p \cdot e^{\prime \prime \prime} \cdots \bar{p}+e^{\prime \prime \prime} \ldots \overline{p+1}}+\ldots e^{\prime \prime \prime} \cdots \overline{\overline{n-1}}}$. Now, if we suppose $e$ to be the greatest error to which we are liable in the measurement of any arc, and each of the succeeding errors to be equal to it, and likewise that $e^{\prime}, e^{\prime \prime}$, 
$\ldots e^{\prime \prime \prime \overline{p-1}}$ are all negative, then it will appear that $\frac{n-p}{n} \times$ $2 p e$ will be the greatest error that can be committed in determining the value of any arc. by adding together the values of the $+p$ ) smaller ares of which it is compounded. For instance, if the interval betwixt the micrometer and the microscope comprehends an arc of $60^{\circ}$, as marked upon the instrument, and this arc is measured against every succeeding arc of $60^{\circ}$ in the whole circle, we shall have the greatest error that can be committed in deducing the arc of $120^{\circ}$ from the ardition of the two first arcs of $60^{\circ}$, equal to $\frac{6-2}{6} \times 2 \times 2 e=2.66 e$. After these remarks, we may proceed to consider how the remaining divisions upon the circle may be examined with the least probable error, and to ascertain the amount of the greatest to which the process can in any case be liable.

Let the arc of $30^{\circ}$ be now measured against every succeeding arc of $30^{\circ}$ in the first, third, fourth, and sixth arcs of $60^{\circ}$, and let the length of each be determined from a separate comparison with the arc of $60^{2}$, in which it is comprehended, and not from a general comparison with all the four. The arc of $15^{\circ}$ must then be measured against every succeeding arc of $15^{\circ}$ in the first, third, fourth, sixth, seventh, ninth, tenth, and twelfth arcs of $30^{\circ}$, and the value of each deduced from a comparison with the arc of $30^{\circ}$, in which it is contained. When this is done, we shall have determined the length of every succeeding arc of $15^{\circ}$, of the first arcs of $30,45,60,75(=60+15), 90$, $105(=90+15), 120(=60+60), 135(=90+45), 150$ $(=120+30), 165(=150+15)$, and $180^{\circ}$ in each semicircle.

We must next measure the arc of $5^{\circ}$ against every suc ceeding arc of $5^{\circ}$ in the whole circle, and deduce the values of the first, and of the sum of the first and second, in each succeeding arc of $15^{\circ}$, from a comparison with the arc of $15^{\circ}$ in which they are contained. We must then proceed to determine the values of the first arc of $3^{\circ}$ in each $15^{\circ}$, and of its multiples the arcs of 6,9 , and $12^{\circ}$. We must also put down the value of the last arc of $3^{\circ}$ in each arc. of $15^{\circ}$, and then deduce the values of the first and last arcs of $1^{\circ}$ in each arc of $15^{\circ}$, from a comparison with the arc of $3^{\circ}$ in which they are respectively contained.

We shall now have measured in each arc of $15^{\circ}$ the first arcs of $1,3,5,6,9,10,12^{\circ}$, and by taking the last arc of one degree, which has likewise been determined, from the 
$\operatorname{arc}$ of $15^{\circ}$, we shall obtain the first arc of $14^{\circ}$. The first $\tau^{\circ}$ of this arc being measured against the second, we ascertain the value of the first $7^{\circ}$; and then, by measuring the first $4^{\circ}$ of the remaining arc of $8^{\circ}$ against the second, we shall get the value of the first $4^{\circ}$, which added to the arc of $\tau^{\circ}$, before determined, will give us the length of the first arc of $11^{\circ}$. The first $2^{\circ}$ of the remaining arc of $4^{\circ}$ must then be measured against the second, and we shall get the value of the fret $2^{0}$, and by adding this are to the arc of $11^{\circ}$, we shall obtain the value of the arc of $13^{\circ}$. By taking away the first arc of $1^{\circ}$ from the arc of $15^{\circ}$, we get the remaining arc of $14^{\circ}$; and then having determined the length of the first $7^{\circ}$ of this arc, by measuring them against the second, we must add it to the arc of $1^{\circ}$, and we shall obtain the arc of $8^{\circ}$. The length of the first $4^{\circ}$ of this arc will then be easily known, by measuring them against the second, as will afterwards that of the first $2^{\circ}$ in the arc of $4^{\circ}$ itself, by measuring them against the second in the same arc.

We have still to ascertain the lengths of all the first arcs of $10,20,30,40$, and 50 minutes contained in each degree, for I shall only consider the case in which the circle is divided into parts of 10 minutes. Now the length of the first arc of $30^{\prime}$ will be obtained by measuring it against the second, and the lengths of the first and second arcs of $20^{\prime}$ (whose sum will give the arc of $40^{\prime}$ ) by measuring the first against each of the remaining ares. The length of the third are of $20^{\prime}$ must likewise be put down, and then the first are of $10^{\prime}$ being measured against the second of the arc of $20^{\prime}$, in which it is included, and also against the two arcs of $10^{\prime}$ contained in the last arc of $20^{\prime}$, its own value, and that of the last $10^{\prime}$ in the degree will be determined from a comparison with the ares of $20^{\prime}$, in which they are respectively comprehended. The length of this last arc of $10^{\prime}$ being taken from that of the whole degree, will give us the length of the first 50', and complete the operation.

In order to ascertain the greatest possible error to which we are liable in the examination, let $\varepsilon$ denote in parts of a second the greatest that can be committed in bisecting any point upon the limb; then, since this error may occur at each end of the arc, it is evident that $e$ in the expression deduced above $\left(\frac{n-p}{n} \times 2 p e\right)$ will become $2 \varepsilon$, and the expression itself $\frac{n-p}{n} \times 4 p \varepsilon$. Hence the possible error will 
be $\frac{2-1}{2} 4 \varepsilon=2 \varepsilon$ at $180^{\circ} ; \frac{2 \varepsilon}{2}+\frac{2-1}{2} \times 4 \varepsilon=3 \varepsilon$ at 9 $\frac{2 \varepsilon}{3}+\frac{3-1}{3} \times 4 \varepsilon=3.33 \varepsilon$ at $60^{\circ} ; \frac{2}{3} \times 2 \varepsilon+\frac{3-2}{3} \times 4 \times$ $2 \varepsilon=4 \varepsilon$ at $120^{\circ}$. The greatest error must therefore lie betwixt 90 and $120^{\circ}$, and nearer to the extremity of the latter than of the former arc. At $105^{\circ}$ it will be 5.50 $\varepsilon$; at $111^{\circ}$ it will be $5 \cdot 50 \varepsilon-\frac{2}{5} \cdot 1,5 \varepsilon+\frac{5-2}{5} \times 4 \times 2 \varepsilon=$ $9,70 \varepsilon$; and at $111^{\circ} 10^{\prime}$ it will be $9,70 \varepsilon-\frac{1}{6} \cdot 1,04 \varepsilon$ (the excess of the error at $111^{\circ}$ above that at $\left.112^{\circ}\right)+3.33 \varepsilon=$ $12.86 \varepsilon$, which will be found to be the greatest error betwixt 105 and $120^{\circ}$, and of course the greatest in the first semicircle. In the other semi-circle, the process being the same, the possible errors must necessarily be the same at the same distances from the first point, reckoning the contrary way upon the circle.

The magnitude of the quantity $\varepsilon$ will of course vary upon circles of the same radius, according to the excellence of the glass employed, and the accuracy of the examiner's eye. It will seldom, however, exceed one second upon a circle whose radius is one foot; and in general it will not amount to so much. I find that I can read off, to a certainty, within less than three fourths of a second; and hence I conclude, that I could examine the divisions of my circle without being liable to a greater error than $9 \cdot 63$ seconds, and those of a circle of three feet radius without the risk of a greater error than 3.21 seconds.

To those people who are accustomed to entertain such exalted notions of the accuracy with which astronomical instruments can with a certainty be divided, this error, I dare say, will appear very considerable; but for my part, I am perfectly satisfied that it bears but a small proportion to the accumulated error which may take place, in spite of the utmost vigilance of the artist, in an instrument divided according to any method which has hitherto been made public. I need not, however, remark upon the very great improbability that the error of examination should ever attain, or approach, to its extreme limit, as this must be sufficiently obvious to any person who is in the least degree conversant with the doctrine of chances; but it may be proper to observe, that we have it in our power (and in this respect the examiner possesses a most important advantage over the divider of an instrument) to diminish its probable amount, as much as we please, by bringing the moveable wires of the micrometer and microscope several times 
times to bisect their respective points in the measurement of every arc, and taking a mean of the different readingsoff for the true position of the wire at the real bisection of the point. The wire may be moved in this manner eight or ten times at each point (if such a degree of caution should be thought necessary) and the mean taken in little more than a minute; so that the time of performing the work will not be so much increased as might perhaps have been apprehended; and when it is completed, we may reasonably presume that the distance of every point from zero (whilst the temperature of the circle continues uniform) will have been determined with sufficient exactness for every practical purpose.

Of the time necessary for the examination, a pretty correct idea may be formed by considering how many measurements are required, and allowing about a minute and a half for each; i.e. a quarter of a minute for bringing the extreme points of the arc to the micrometer and the microscope, and a minute and a quarter for making the several bisections. Now, in dividing the whole circle into arcs of $15^{\circ}$ each, it will appear that forty-four measurements must be performed; and to examine every point in each $\operatorname{arc}$ of $15^{\circ}$, there will be 161 required, making in all 3908 measurements ; and consequently the time necessary for completing the whole work will be 5862 minutes, or about 98 hours.

The time and lahour required for this examination are, no doubt, very considerable; but it ought to be recollected, that it will render any great degree of precision, in dividing the instrument, totally unnecessary. Whoever indeed employs this method of examination, will be virtually the divider of his own instrument; and all that he will ask of the artist, is to make him a point about the end of every five or ten minites, whose distance from zero he will determine for himself, and enter in his book to be referred to when wauted. We may likewise observe, that by this examination we shall not only be secured against the errors of division, but against those which arise from bad centering, and from the imperfect figure of the circle, and which in general are of too great a magnitude to be neglected.

It will, I dare say, bave occurred to you, that whenever we are desirous that an observation should, be particularly exact, we may guard it against the effects of unequal expansion or contraction in the metal, by means of the apparatus which I have described : for we have only to measure the arc which has been determined by the obstrvation 
against the whole circle, or against the multiple of it, which approaches nearest to the circle, and from thence to deduce its value in the manner explained above, and we shall either have entirely excluded the error which we apprebended, or have rendered it too small to be of any importance. Suppose, for instance, that the arc determined by the observation was $48^{\circ}$; then by measuring it against the whole circumference increased by an arc of $24^{\circ}$, we shall obtain a result free from any greater error of unequal temperature, than one eighth of the increase or decrease of this arc of $24^{\circ}$ beyond a due proportion to that of the circle itself.

This expedient gives us all the advantages of the French circle of repetition, without the inconvenience arising from being obliged to turn the instrument, and move the telescope, so many times in the course of the observation. Nay, I am persuaded that the result may be made more accurate in this way than by the French method, because not only can the object be more frequently observed, but the contacts or bisections, it may be presumed, will be more exact when the observer is not disturbed by the hurry attendant upon the use of the repeating circle; and with respect to any error in the instrument, from whatever cause it may arise, it will be as effectually excluded by the process which I recommend, as by moving the telescope round the circle. Besides, this method is applicable either to the azimuth or altitude circle, or indeed to any circle which turns upon its own axis; whereas the French method can never be applied to the azimuth circle, nor to any other circle which does not turn both upon its own axis and upon one which is perpendicular to it.

After all, however, it is possible that the process which I have been explaining to you may be no new discovery, and that you may be already acquainted with it. If this should be the case, you will be kind enough to inform me. At any rate, indeed, I should esteem myself greatly obliged, if you would favour me with your sentiments upon the subject, as soon as you can do it with perfect convenience to jourself.

I am, dear sir, yours, \&c.

William Lax. 